\title{
MALDI-TOF mass spectrometry-based direct-on-target microdroplet growth assay: a novel assay for susceptibility testing and beyond
}

\author{
loannis K Neonakis*,1 \& Demetrios A Spandidos ${ }^{2}$ \\ ${ }^{1}$ Department of Clinical Microbiology \& Microbial Pathogenesis, University Hospital of Heraklion, Crete 71201, Greece \\ ${ }^{2}$ Laboratory of Clinical Virology, School of Medicine, University of Crete, Crete 71003, Greece \\ *Author for correspondence: Tel.: +30 694242 2527; ineonakis@gmail.com
}

\begin{abstract}
"Combined with the ability of MALDI-TOF MS for species identification, it can provide valuable information regarding critical clinical samples, such as positive blood cultures within a short period of time, markedly affecting the patient management."
\end{abstract}

First draft submitted: 10 April 2019; Accepted for publication: 29 April 2019; Published online: 4 July 2019

Keywords: antimicrobial susceptibility testing • direct-on-target microdroplet growth assay • MALDI-TOF mass spectrometry • minimum inhibitory concentration • multidrug resistant microorganisms

The timely detection of resistant isolates in clinical samples is a critical issue for patient management. Over the past few years, the application of matrix-assisted laser desorption ionization-time of flight mass spectrometry (MALDI-TOF MS) has provided a significant contribution to this field. Depending on the principle applied, there are variations to the methodology. One approach is the comparison of the MS profiles obtained for an index antibiotic prior to and after its incubation with the strain tested [1,2]. Alterations in its MS profile are indicative of a hydrolytic enzyme production. Another approach is the detection of specific MS peaks highly correlated directly or indirectly, with the production of certain enzymes, conferring resistance. Thus, the presence of an 11,109 MS peak in the spectra of Gram-negative isolates is indirectly associated with KPC production by the isolate [3], whereas the presence of a 25,544 MS-peak is directly associated with a KPC production [4].

A very promising novel approach in detecting microbial resistance using MALDI-TOF has recently been proposed [5-7]. Apart from its simplicity, speed and practicality, MALDI-TOF MS-based direct-on-target microdroplet growth assay (DOT-MGA) has the great advantage of generalizability. It can provide valuable information regarding susceptibilities thus revealing the underlying resistance mechanisms.

\section{The method}

The principle of this methodology [5-7] is as follows: the microorganism is incubated for a short period of time (3-5 h) with and without (growth control) the index antibiotic in nutrient broth as microdroplets directly on MALDI-TOF MS target spots. An antibiotic concentration gradient can be achieved in a series of consecutive spots. In order to avoid microdroplet evaporation, the target is incubated in a simple plastic transport box used as a humidity chamber, with addition of water onto the box bottom. Following the incubation period, the broth is separated from microbial cells by contacting the microdroplets with an absorptive material. A test is considered valid if MALDI-TOF can properly identify the microorganism in growth control. In parallel, the achievement or not of microorganism identification by MALDI-TOF in each spot with the different specific concentration of the index antibiotic reveals the presence or absence of the microorganism growth in each particular spot. Since the exact concentration of the antibiotic in each droplet spot is known, the antibiotic minimum inhibitory concentration (MIC) can be evaluated and the microorganism can be characterized as susceptible or not, pertaining to the certain antibiotic. By using this assay with a panel of different antibiotics, not only can the susceptibility status of an isolate be determined, but the underlying resistance mechanisms can also be revealed.

Future Medicine 
In their initial proof-of-principle study, Idelevich et al. provided solutions to many technical challenges and issues, such as the optimum microdroplet volume, the problem of microdroplet evaporation during incubation, the optimum incubation time, the possible usefulness of on-target protein extraction as well as the option of incubation in microtitrate plates and subsequent transfer on to target spots [5]. The study included 24 Klebsiella pneumoniae and 24 Pseudomonas aeruginosa clinical isolates. In each group, there were 12 meropenem-susceptible and 12 meropenem-nonsusceptible consecutive, nonduplicate isolates. Meropenem was selected as the index antibiotic in order to assess the ability of the method to detect major resistance mechanisms. $K$. pneumoniae was selected as a representative of Enterobacteriaceae and $P$. aeruginosa since it is considered a critical multidrug-resistant microorganism. Emphasis was given to the simplicity of the steps so that the method could be easily executed by users. Furthermore, a reliable algorithm for data analysis was suggested. MALDI-TOF MS was performed with standard protocol using a MALDI Biotyper system (Bruker Daltonik). The standard initial inoculum size for antimicrobial susceptibility testing (AST) recommended by the Clinical Laboratory Standards Institute (CLSI) [8] and International Organization for Standardization (ISO) [9] guidelines was used in the experiments. No fast on-target protein extraction, such as the addition of formic acid on dried spots, was used although the authors suggested that such an addition may be helpful for the AST of Gram-positive bacteria or yeasts. For the detection of carbapenem nonsusceptibility in K. pneumoniae, optimum results (100\% validity, $100 \%$ sensitivity and $100 \%$ specificity) were obtained using a $6 \mu \mathrm{l}$ droplet volume with $4 \mathrm{~h}$ of incubation, whereas for $P$. aeruginosa, optimum results $(83.3 \%$ validity, $100 \%$ sensitivity and $100 \%$ specificity) were obtained with the same droplet volume and an incubation period of $5 \mathrm{~h}$.

The same research group tried to implement the DOT-MGA assay directly on positive blood cultures (BCs) [6]. They evaluated the optimum method for positive BC broth processing by comparing a total of four methods: two dilution methods (which are generally performed with minimum hands-on time and are suitable for robotized automation) and two centrifugation methods (which require a technician's attention, but can provide a clean microbial pellet) [6]. The study included 14 consecutive Enterobacteriaceae of various species determined as meropenem nonsusceptible by phenotypic AST and 14 consecutive Enterobacteriaceae of the same species distribution that were meropenem susceptible. For the two dilution methods, it was assumed that the bacterial concentration in positive-BC broth was approximately $10^{8} \mathrm{CFU} / \mathrm{ml}$ and proper filtrate dilutions were made to prepare the inoculum, whereas for the centrifugation methods, the resuspended pellet was standardized to a $0.5 \mathrm{McFarland}$ standard turbidity and following appropriate dilutions, the inoculum size proposed by the CLSI [8] and ISO [9] guidelines for AST was achieved. In parallel, the final meropenem breakpoint concentration of $2 \mu \mathrm{g} / \mathrm{ml}$ differentiating between susceptible and nonsusceptible Enterobacteriaceae as proposed by the European Committee on Antimicrobial Susceptibility Testing (EUCAST) was established [10]. For each sample, there was also a growth control, a droplet with no antibiotic. Subsequently, following an incubation period (both 3- and 4-h periods were evaluated) the MALDI-TOF MS DOT-MGA was performed. If the MALDI provided a proper species identification $(\geq 1.7)$ for the growth control, then the test was considered valid and the result of the droplet with the presence of antibiotic was taken into account. In this droplet containing the antibiotic, if indeed a valid species identification was obtained by MALDI-TOF (score, $\geq 1.7$ ), then the strain was considered nonsusceptible, whereas if there was no identification, then the strain was considered susceptible. Optimal results (96.3\% validity, $91.7 \%$ sensitivity and $100 \%$ specificity) were obtained with the lysis/centrifugation method using a 4-h incubation period.

Correa-Martínez et al. proposed a very rapid assay (with only 4-h incubation period) for extended-spectrum $\beta$-Lactamases (ESBL) and AmpC screening among Enterobacteriaceae [7]; the study included 50 Enterobacteriaceae. With the use of the DOT-MGA methodology and by achieving a gradient of antibiotic concentrations in consecutive target spots, they managed to determine the MICs not only for four key cephalosporins (cefpodoxime [CPD]; ceftazidime [CAZ]; cefotaxime [CTX]; cefepime [CFP]), but also for four combinations of cephalosporines with appropriate inhibitors (clavulanic acid [CA]; cloxacillin [CLX]) namely: CAZ+CA, CTX+CA, CFP+CA and CTX+CA+CLX [7]; this was done for each isolate. Subsequently, by applying the criteria and algorithm suggested by EUCAST [11], it was easy to reveal not only the susceptibility status of the isolate, but also the underlying resistance mechanisms. Comparing the results with those of polymerase chain reaction, the positive and negative percent agreement values (PPA and NPA) were 94.4 and 100\% for ESBL, 94.4 and $93.8 \%$ for AmpC and 100 and $100 \%$ for $\mathrm{ESBL}+\mathrm{AmpC}$, respectively.

The DOT-MGA methodology is a very promising one as it is rapid, simple in principle, practicable and significantly expands the applicability of MALDI-TOF MS in the field of antibiotic susceptibility testing. Combined with the ability of MALDI-TOF MS for species identification, it can provide valuable information regarding 
critical clinical samples, such as positive blood cultures within a short period of time, markedly affecting the patient management. If the identification of a microorganism and its resistance status are readily available within only a few hours after a blood culture is signaled as positive, this will markedly alter the clinical and epidemiological decisions drawn.

This is a novel methodology and possible occurring technical issues regarding standardization or reproducibility should be resolved. On the other hand, if this methodology will be combined with additional technical traits such as automation, advanced software or addition of various lyophilized substances to the spots, its potentials will be greatly expanded and the value of its contribution to the field of rapid susceptibility testing and beyond, will be enormous.

Large-scale studies validating the methodology are strongly advocated and highly anticipated.

\section{Financial \& competing interest's disclosure}

The authors have no relevant affiliations or financial involvement with any organization or entity with a financial interest in or financial conflict with the subject matter or materials discussed in the manuscript. This includes employment, consultancies, honoraria, stock ownership or options, expert testimony, grants or patents received or pending, or royalties.

No writing assistance was utilized in the production of this manuscript.

\section{References}

1. Hrabák J, Walková R, Studentová V et al. Carbapenemase activity detection by matrix-assisted laser desorption ionization-time of flight mass spectrometry. J. Clin. Microbiol. 49(9), 3222-3227 (2011).

2. Burckhardt I, Zimmermann S. Using matrix-assisted laser desorption ionization-time of flight mass spectrometry to detect carbapenem resistance within 1 to 2.5 hours. J. Clin. Microbiol. 49(9), 3321-3324 (2011).

3. Lau AF, Wang H, Weingarten RA et al. A rapid matrix-assisted laser desorption ionization-time of flight mass spectrometry-based method for single-plasmid tracking in an outbreak of carbapenem-resistant Enterobacteriaceae. J. Clin. Microbiol. 52(8), 2804-2812 (2014).

4. Figueroa-Espinosa R, Costa A, Cejas D et al. MALDI-TOF MS based procedure to detect KPC-2 directly from positive blood culture bottles and colonies. J. Microbiol. Methods pii:S0167-7012(18)30920-5 (2019) (Epub ahead of print).

5. Idelevich EA, Sparbier K, Kostrzewa M, Becker K. Rapid detection of antibiotic resistance by MALDI-TOF mass spectrometry using a novel direct-on-target microdroplet growth assay. Clin. Microbiol. Infect. 24(7), 738-743 (2018).

6. Idelevich EA, Storck LM, Sparbier K et al. Rapid direct susceptibility testing from positive blood cultures by the matrix-assisted laser desorption ionization-time of flight mass spectrometry-based direct-on-target microdroplet growth assay. J. Clin. Microbiol. 56(10), e00913-18 (2018).

7. Correa-Martínez CL, Idelevich EA, Sparbier K et al. Rapid detection of extended-spectrum $\beta$-lactamases (ESBL) and AmpC $\beta$-lactamases in enterobacterales: development of a screening panel using the MALDI-TOF MS-based direct-on-target microdroplet growth assay. Front. Microbiol. doi:10.3389/fmicb.2019.00013 (2019) (Epub ahead of print).

8. Clinical Laboratory Standards Institute. Methods for dilution antimicrobial susceptibility tests for bacteria that grow aerobically; approved standard. M07-A10 (10th Edition), PA, USA (2015).

9. ISO. 20776-1. Clinical laboratory testing and in vitro diagnostic test systems- susceptibility testing of infectious agents and evaluation of performance of antimicrobial susceptibility test devices-Part 1: reference method for testing the in vitro activity of antimicrobial agents against rapidly growing aerobic bacteria involved in infectious diseases. International Organization for Standardization. Geneva, Switzerland (2006).

10. EUCAST. Breakpoint tables for interpretation of MICs and zone diameters, version 7.1 (2017). www.eucast.org/fileadmin/src/media/PDFs/EUCAST_files/Breakpoint_tables/v_7.1_Breakpoint_Tables.pdf

11. EUCAST Guidelines for Detection of Resistance Mechanisms and Specific Resistances of Clinical and/or Epidemiological Importance The European Committee on Antimicrobial Susceptibility Testing. Version 2.0 (2017). www.eucast.org 
